\title{
Role of MRI in Evaluation of Ring Enhancing Lesions of Brain in Correlation with Mr Spectroscopy
}

\author{
R. Archana ${ }^{1}$, P. Sunil Kumar ${ }^{2}$, Anurudh Kishore ${ }^{3}$ \\ ${ }^{1}$ Associate Professor, ${ }^{2}$ Assistant Professor, ${ }^{3}$ Post Graduate, Department of Radiodiagnosis: Osmania Medical College, \\ Hyderabad, Telangana, India.
}

Corresponding author: Dr. P. Sunil Kumar, Assistant Professor, Department of Radiodiagnosis, Osmania Medical College, Hyderabad, Telangana, India.

DOI: http://dx.doi.org/10.21276/ijcmsr.2018.3.4.9

How to cite this article: RR. Archana, P. Sunil Kumar, Anurudh Kishore. Role of MRI in evaluation of ring enhancing lesions of brain in correlation with $\mathrm{mr}$ spectroscopy. International Journal of Contemporary Medicine Surgery and Radiology. 2018;3(4):D33-D37.

\section{A B S T R A C T}

Introduction: On neuroimaging, the most common abnormality is the multiple ring enhancing lesions of the brain. This study was intended to characterize imaging findings in various ring enhancing lesions of brain.

Material and Methods: 40 patients (Males=28; Females=12) were evaluated in this study conducted at Osmania General Hospital and MNJIO and RCC over a period of 2 years. MRI along with MRS was performed in patients ranging from ages of 7-66 years.

Results: Out of the 40 patients evaluated 18 cases were tuberculomas, 10 were NCC, 4 abscesses, 4 metastases, 2 cases of primary brain tumors and 2 cases of toxoplasmosis. The highest incidence of REL's were found in 21-30 years age group accounting for 10 cases and least was seen in age group of $>60$ years constituting $5 \%$. 28(70\%) were males and 12 (30\%) were females. $14(35 \%)$ were noted on the right side, 10 (25\%) were noted on the left side, Seizures are the most common presenting complaint in 50\% of cases. 14 (35\%) of them presented with a single lesion. Majority 28 (70\%) of them showed RELs $<2 \mathrm{~cm}$. Choline peak was observed in 15 cases, Lipid in 18 cases, Lactate in 22 cases, reduced NAA peak in 7 cases and amino acids in 3 cases.

Conclusion: The most sensitive modality which can characterize the intracranial ring enhancing lesions RELs is MRI's. Based on characteristic imaging findings, MRI plays a pivotal role in patient management by suggesting the correct diagnosis.

Keywords: Magnetic Resonance Imaging, Lesions.

Key words: MRI, Ring Enhancing Lesions, Brain

\section{INTRODUCTION}

In neuro-imaging, the most common abnormalitiy faced is multiple ring-enhancing lesions. The advanced new imaging techniques which are widely used to detect the lesions are computed tomography and magnetic resonance imaging (MRI). Cerebral multiple ring-enhancing lesions are the wide range of etiologies which may be present. ${ }^{1,2}$ On using non-contrast computed tomography studies, these lesions may appear as hypodense or isodense mass lesions in neuroimaging. Within the hypodensity region, there is a ring- or a homogeneous disk-like enhancement after contrast administration. By a varying amount of perifocal vasogenic edema, these are surrounded and are enhancing lesions of variable size. The ring-enhancing lesions are located at the junction of the gray and white matter and are located in the sub-cortical area, deep in the brain parenchyma or may even be superficial. ${ }^{3}$ Clinically advantageous in early detection of disease are MRI's and these are visually demonstrated as unmistakable contrast between gray and white matter and tumor ischemia/infarct, edema, MS plaques, infection/ abscess and hemorrhage. Inherent sensitivity of MRI's as well as its capability to directly image in any plane without reformatting, and to be unimpeded or undistorted by bony structures are the contributing factors. MR spectroscopy plays a pivotal tool in differential diagnosis between brain abscesses and non-infectious lesions such as primary brain tumor, lymphoma, brain metastasis, and tuberculoma. The extent and nature of changes on a routine MRI scan is possible by magnetic resonance spectroscopy (MRS) and it provides information by analyzing the presence or ratio of tissue metabolites such as NAA, creatine, choline, and lactate. To detect functional metabolic changes, usage of faster MRS applications widely with higher signal-tonoise ratio (SNR) and spatial resolution allows to provide more data to understand the exact nature of the tumour and the morphological and physiological changes occurring in the surrounding brain parenchyma. HMRS is useful in monitoring disease progression and treatment effects which is demonstrated by longitudinal studies. MR spectroscopy plays an important prognostic implication. ${ }^{3}$ This study was intended to characterize imaging findings in various ring enhancing lesions of brain. 


\section{MATERIAL AND METHODS}

It was a prospective descriptive study in patients from Osmania general hospital and teaching hospitals of Osmania medical college MNJIO and RCC and Niloufer hospital. All patients referred to the department of Radio diagnosis with clinically suspected ring enhancing lesions of brain in a period of december 2015 to November 2017. Inclusion Criteria were all ring enhancing lesions of brain detected on contrast MR studies are taken up and cases of all age groups irrespective of sex. Exclusion Criteria were patients who had history of claustrophobia, patients having history of metallic implants insertion, cardiac pacemakers and metallic foreign body, allergic to gadolinium based contrast agents and patients with poor kidney function (GFR $<30 \mathrm{ml} / \mathrm{min} / 1.73 \mathrm{~m} 2$ ). The MRI scan was performed on MR PHILIPS ACHIEVA $1.5 \mathrm{~T}$ and GE Signa HDxt 1.5 T. PHILIPS ACHIEVA possesses an Ultra-compact, Superconducting, Active shielded superconducting magnet with a magnetic field strength of $1.5 \mathrm{~T}$. SENSE coils was used for acquisition of images.

\section{STATISTICAL ANALYSIS}

Statistical analysis was performed using percentages and proportions.

\section{RESULTS}

Total 40 patients presented with various ring enhancing lesions. Tuberculomas (45\%) was the most common pathology followed by NCC (25\%), Abscesses (10\%), metastasis (10\%), primary brain tumour (5\%) and toxoplasmosis (5\%).

The highest incidence of REL's were found in 21-30 years age group accounting for 10 cases and least was seen in age

\begin{tabular}{|l|c|c|}
\hline Age (In Years) & No. of Cases & Percentage \\
\hline $0-10$ & 6 & 15 \\
\hline $11 .-20$ & 8 & 20 \\
\hline $21-30$ & 10 & 25 \\
\hline $31-40$ & 4 & 10 \\
\hline $41-50$ & 4 & 10 \\
\hline $51-60$ & 6 & 15 \\
\hline$>60$ & 2 & 5 \\
\hline Gender & & \\
\hline Male & 28 & 70 \\
\hline Female & 12 & 30 \\
\hline Side of pathology & 14 & 35 \\
\hline Right & 10 & 25 \\
\hline Left & 14 & 35 \\
\hline Bilateral & 2 & 5 \\
\hline Midline & & \\
\hline Symptom & 20 & 50 \\
\hline Seizures & 8 & 20 \\
\hline Headache & 5 & 12 \\
\hline Vomiting & 3 & 8 \\
\hline Weakness & 2 & 5 \\
\hline Fever & 2 & 5 \\
\hline Ataxia & & \\
\hline & & \\
\hline
\end{tabular}

\begin{tabular}{|l|c|c|}
\hline Number of Lesions & Number of Cases & Percentage \\
\hline 1 & 14 & 35 \\
\hline $2-4$ & 18 & 45 \\
\hline$>4$ & 8 & 20 \\
\hline Size of Lesion (In Cms) & & \\
\hline$<2$ & 28 & 70 \\
\hline $2-4$ & 8 & 20 \\
\hline$>4$ & 4 & 10 \\
\hline \multicolumn{2}{|c|}{ Table-2: Number of Ring Enhancing Lesions in a Patient } \\
\hline
\end{tabular}

\begin{tabular}{|l|c|c|}
\hline Metabolite Peak & Number of Cases & Percentage \\
\hline Choline & 15 & 37.5 \\
\hline Lipid & 18 & 45 \\
\hline Lactate & 22 & 55 \\
\hline Reduced NAA & 7 & 17.5 \\
\hline Amino Acids & 3 & 7.5 \\
\hline \multicolumn{2}{|c|}{ Table-3: List of Various Metabolite Peaks Noted in Various } \\
\multicolumn{2}{|c|}{ Enhancing Lesions } \\
\hline
\end{tabular}
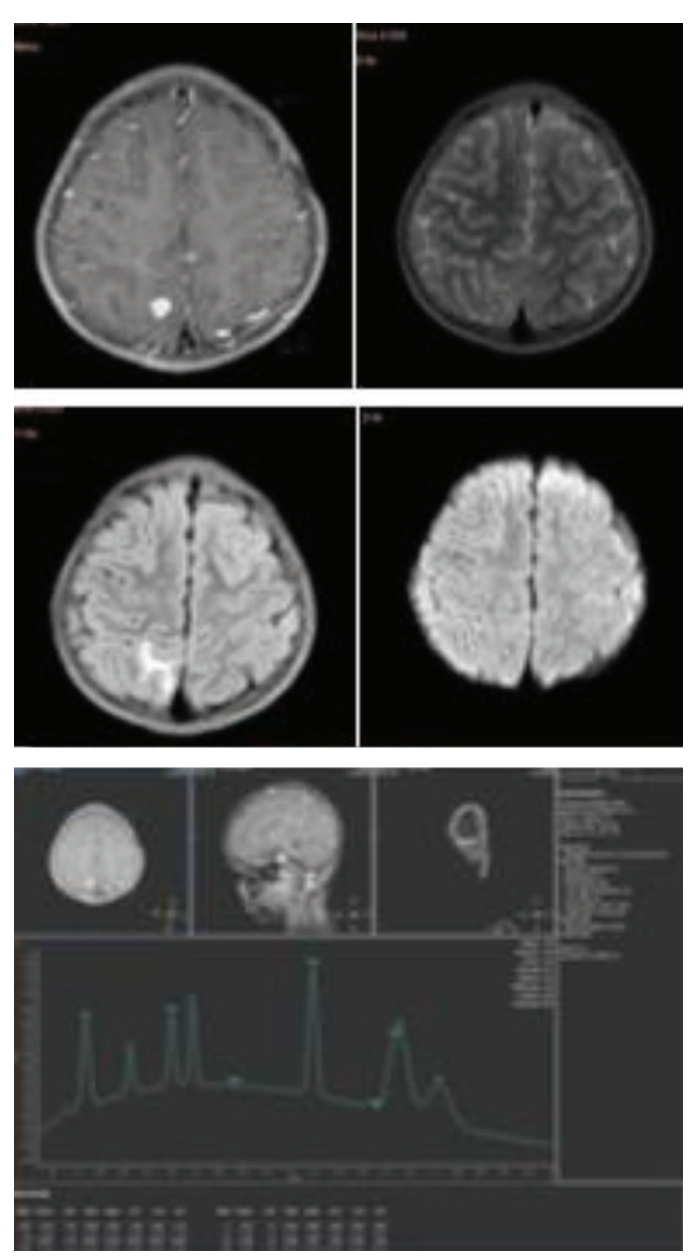

Figure-1: T2 FLAIR hyperintense lesion with hypointense rim showing restriction on DWI with lipid and lactate peak on MRS in right parietal region. S/O Tuberculoma (case-1).

group of $>60$ years constituting $5 \% .28(70 \%)$ were males and $12(30 \%)$ were females. $14(35 \%)$ were noted on the right side, $10(25 \%)$ were noted on the left side, Seizures are the most common presenting complaint in $50 \%$ of cases (table-1,2). $14(35 \%)$ of them presented with a single lesion. Majority 28 

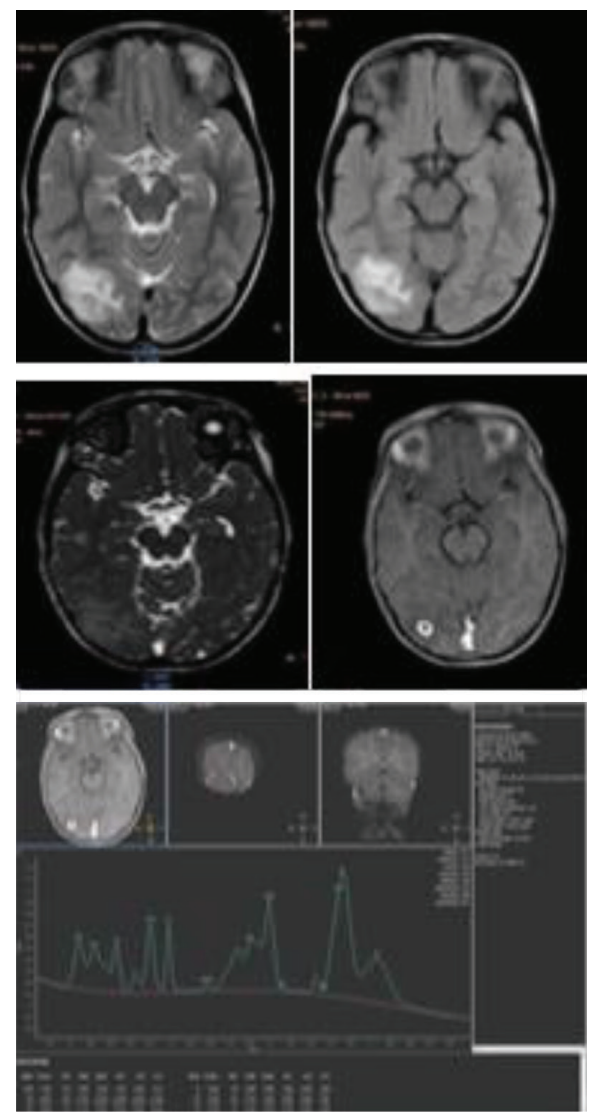

Figure-2: T2 FLAIR hyperintense lesion with hypointense rim, showing hypointense scolex on FIESTA with lipid, lactate peak on MRS S/O NCC (case-2).
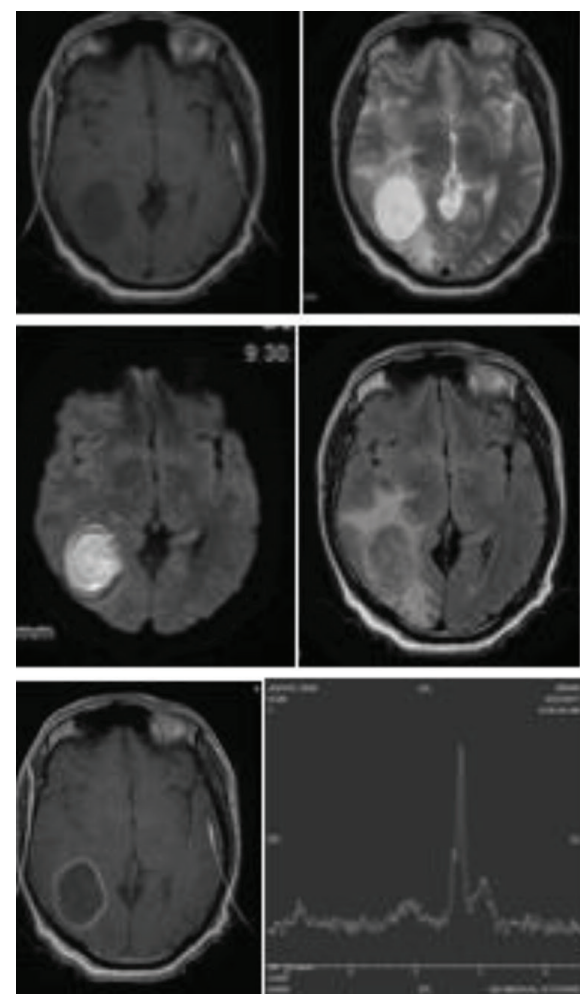

Figure-3: Shows restriction on DWI with adjacent perilesional edema noted in right parieto- occipital region with lipid, lactate peak on MRS S/O PYOGENIC ABSCESS in T1 hypointense, T2 FLAIR hyperintense lesion with hypointense rim (case-3).
(70\%) of them showed RELs < $2 \mathrm{~cm}$. Evaluated spectroscopy was possible only 35 cases and was not performed in 5 cases because of presence of the lesion close to the bone. Choline peak was observed in 15 cases, Lipid in 18 cases, Lactate in 22 cases, reduced NAA peak in 7 cases and amino acids in 3 cases.

\section{DISCUSSION}

The lesion is accurately demonstrated by magnetic resonance imaging which is a noninvasive, multiplanar and highly accurate method with better inherent contrast. In various ring enhancing lesions, MRI helps in accurately assessing the changes in brain and for accurately diagnosing and introducing treatment immediately. The present study was a prospective study done in the Department of Radiodiagnosis and Imaging, Osmania medical college aimed at studying the MR appearances in various ring enhancing lesions of the brain. In our study, 40 patients were evaluated whose age group ranged from 7 to 66 years. The highest incidence of REL's were found in 21-30 years age group accounting for $25 \%$ of cases and least was seen in age group of $>60$ years constituting 5\%. 24(60\%) were males and 16 (40\%) were females. Seizures are the most common presenting complaint in $70 \%$ of cases. Headache $(30 \%)$, fever $(10 \%)$, vomiting (25\%), ataxia (5\%) and motor weakness (10\%) were the other presenting complaints. Tuberculomas (45\%) is the most common pathology followed by NCC (25\%), Abscesses (10\%), metastasis (10\%), primary brain tumour (5\%) and toxoplasmosis (5\%). In a study conducted by Schwartz et $\mathrm{al}^{4} 40 \%$ cases were gliomas. In India, the higher incidence of tuberculomas is because of the higher prevalence of tuberculosis. Among the forty patients with RELs on the right side, 14(35\%) were noted, on the left side, 10 (25\%) were noted, bilaterally, $14(35 \%)$ were seen and in the midline, 2 (5\%) were observed. Forty patients were evaluated, out of which, single lesion was seen in 14 (35\%) patients. In 18 (45\%) of cases, 2-4 lesions were noted and > 4 RELs were seen in $8(20 \%)$ of cases. Majority $28(70 \%)$ of them showed RELs $<2 \mathrm{~cm}$, lesions of sizes between $2-4 \mathrm{~cm}$ was seen in 8 (20\%) and only in $4(10 \%)$ lesions size is greater than $4 \mathrm{~cm}$. Size of the maximum number of lesions which were falling in one category were considered in case of multiple lesions. In present study evaluated spectroscopy was possible in only 35 cases and was not performed in 5 cases because of presence of the lesion close to the bone. In 15 cases, Choline peak was observed, Lipid in 18 cases, Lactate in 22 cases, reduced NAA peak in 7 cases and amino acids in 3 cases. In 18(45\%) of cases, tuberculomas were seen. Among the 18 cases (males $=13$ : females $=5$ ). Single lesions were observed in 5 cases (27.8\%) and multiple in 13 cases (72.2\%). Single lesions were observed as conglomerate lesions which are hypointense on both T1 and T2. They show an iso to hyperintense ring on $\mathrm{T} 1$ weighted images which was seen in 10 cases in our study. They may show partial or complete restriction which can be seen in 14 cases (77.7\%). A nodular or irregular ring like enhancement is shown by the lesions. All our cases presented with ring like enhancement. In addition to the ring enhancing lesions, in 2 cases, nodular enhancement can also be seen. In 12(66.6\%) cases, MRS showed a lipid peak and it 
plays a pivotal role in tuberculomas identification from other infective granulomas. The tuberculoma can also be identified on MRI with the help of T2 weighted images whether the stage of the tuberculoma is caseous or non caseous. In identifying the size of the tuberculomas, post contrast images are very helpful because of its excellent spatial resolution and its ability to differentiate the granuloma from its surrounding edema. Tae Kyoung Kim et $\mathrm{al}^{5}$ showed that the granulomas showed a slightly hyperintense rim on T1-weighted images. The entire portion of the granuloma showed slightly heterogeneous isointensity or hypointensity with small markedly hypointense foci on T2-weighted images. There were single or multiple conglomerate ring enhancements within a tuberculoma on postcontrast T1-weighted images in all six patients. Jayasundar $\mathrm{R}$ et $\mathrm{al}^{6}$ concluded that for differentiating tuberculomas from both non-specific IG and NCC, presence of lipid can be used. Neurocysticercosis was seen in 10 (males $=6$; females $=4$ ) cases, out of fourty patients evaluated. 4 patients were presented with single lesions whereas 6 patients were presented with multiple lesions. All the cases were showing intraparenchymal forms of NCC and in 2 cases, subarachnoid cysticercosis was seen. In 4 cases, using Balanced FFE/FIESTA sequence, scolex was identified. From MRS, choline peak was shown and reduced NAA peak. In identifying calcified lesions which were seen in 3 cases (30\%), gradient echo imaging played a significant role. On T1 weighted images, all the lesions were hypo to isointense and hyperintense. Inversion on FLAIR was shown by 4 lesions suggesting that the contents are similar to that of CSF. In all cases suggestive of active lesions, intense ring enhancement with surrounding perilesional edema was seen. In our study, a single case of intraventricular cysticercosis was not seen probably because of the small sample of study. In 22\% of cases Martinez et al reported intraventricular neurocysticercosis. ${ }^{7}$ Parenchymal cysticercosis was better identified on MRI than CT in our study as compared to the study done by Suss Ra et al. ${ }^{8}$ Features of parenchymal forms of NCC in our study are similar to the study done by Amaral LL et al. ${ }^{9}$ Cho / Cr ratio was less than 1.1 in all NCC and more than 1.2 in all tuberculoma which is similar to the study performed by Kumar et $\mathrm{al}^{10}$ and Jayasunder et al. ${ }^{6} \mathrm{In}$ present study abscess were found in 4 cases i.e. $10 \%$ (males 2; females $=2$ ). In 3 cases (40\%), single abscess was found whereas one case had multiple abscesses. In all the cases, sizes $>2 \mathrm{~cm}$ were shown and one case was $>4 \mathrm{~cm}$. On T1 weighted images, all were hypointense with a hyperintense rim noted in 3 patients and were hyperintense on T2 weighted images with a surrounding hypointense rim (3 cases). They showed complete diffusion restriction and lactate peak in all 4 cases was shown by MRS suggesting anaerobic glycolysis. In Halmes et al, the appearance of abscesses on MR was observed. We correlated our findings with those described and distinguished the peripheral oedema, central necrosis and the characteristic pattern of peripheral enhancement of the abscess capsule. ${ }^{11}$ Our findings were similar to the study conducted by Shukla-Dave A et a ${ }^{12}$ and Leuthardt EC et al. ${ }^{13}$ In our study 4 cases were metastasis (males $=1$; females $=3$ ), in all the four cases, multiple lesions were identified. All the cases showed high Cho / Cr and Cho / NAA levels.
On T2, all 4 cases were hyperintense with 2 cases showing inversion on FLAIR suggesting cystic metastasis. Primary was identified in three cases in breast, lung. After contrast administration, thick, irregular type of ring enhancement was noted. Our findings were similar to the study conducted by Vieth $R G$ et ${ }^{11}{ }^{14} 2$ cases were toxoplasmosis (males $=1$; females $=1$ ), out of the 40 patients, multiple lesions were identified in both the cases. In both cases, T1,T2 hyperintense lesions with peripheral restriction on DWI with lactate peaks were shown on MRS. 2 cases were having primary brain tumors (glioblastoma multiformae) in both the cases the lesions were hypointense on T1,Hyperintense on T2, showing choline peak on MRS with reduced NAA level, out of the 40 patients. The lesion was crossing the midline through corpus callosum in one case.

\section{CONCLUSION}

The most sensitive modality which can characterize the intracranial ring enhancing lesions RELs is MRI's. To differentiate between benign and malignant lesions, hypointensity on T2 with partial or complete restriction on DWI images and lipid peak on MRS, pattern of signal intensity on T2 and FLAIR, DWI and MRS is more in favour of Tuberculoma. NCC is suggested by hyperintensity on T2 with no diffusion restriction, presence of scolex on Balanced FFE/ FIESTA. Abscesses show a hypointense rim on T2 with complete diffusion restriction. MRS shows Lactate and Amino Acids. MRI being non invasive and nonradiating is an ideal imaging modality.

\section{REFERENCES}

1. Omuro AM, Leite CC, Mokhtari K, Delattre JY. Pitfalls in the diagnosis of brain tumours. Lancet Neurol 2006;5(1):937-48.

2. Cunliffe CH, Fischer I, Monoky D, Law M, Revercomb $\mathrm{C}$, Elrich $\mathrm{S}$, et al. Intracranial lesions mimicking neoplasms. Arch Pathol Lab Med 2009;133(3):101-23.

3. Bulakbasi N. Clinical applications of proton MR spectroscopy in the diagnosis of brain tumours. Spectroscopy 2004; 18(2):143-153.

4. Schwartz KM, Erickson BJ, Lucchinetti C Pattern of T2 hypointensity associated with ring- nhancing brain lesions can help to differentiate pathology Neuroradiology. 2006;48(3):143-162

5. Tae Kyoung Kim et al Intracranial Tuberculoma: Comparison of MR with Pathologic Findings AJNR 16:1903-1908

6. Jayasundar R, Singh VP, Raghunathan P, Jain K, Banerji AK Inflammatory granulomas: evaluation with proton MRS NMR Biomed. 1999 May;12(3):139-44.

7. HR Martinez,R Rangel- Guerra, G Elizondo, J Gonzalez, LE Todd, J Ancer, and SS Prakash MR Imaging in neurocysticercosis: a study of 56 cases AJNR 1989; 10 (5): 1011-1019

8. Suss RA, Maravilla KR, Thompson J MR imaging of intracranial cysticercosis: comparison with $\mathrm{CT}$ and anatomopathologic features. AJNR AmJNeuroradiol. 1986;7(2):235-42.

9. Amaral L,Maschietto M,Maschietto R, Cury R, Ferreira NF, Mendonça R, Lima SS. Ununsual manifestations of 
neurocysticercosis in MR imaging: analysis of 172 cases Arq Neuropsiquiatr. 2003;61(3A):533.

10. Kumar A, Kaushik S, Tripathi RP, Kaur P, Khushu $\mathrm{S}$. Role of in vivo proton MR spectroscopy in the evaluation of adult brain lesions: Our preliminary experience. Neurol India 2003; 51(6): 474-478.

11. Halmes AB, Zimmerman RD, Morgello S,Weingarten $\mathrm{K}$, Becker RD, Jennis R, Deck MD. MR Imaging of brain abscesses. AJR 1989;152 (5):1073-85

12. Shukla-Dave A, Gupta RK, Roy R, Husain N, Paul L, Venkatesh SK, Rashid MR, Chhabra DK, Husain $\mathrm{M}$ Prospective evaluation of in vivo proton $\mathrm{MR}$ spectroscopy in differentiation of similar appearing intracranial cystic lesions. Magn Reson Imaging. 2001;19(1):103-10.

13. Leuthardt EC, Wippold FJ 2nd, Oswood MC, Rich KM. Diffusionweighted MR imaging in the preoperative assessment of brain abscesses. Surg Neurol. 2002;58(6):395-402;

14. Vieth RG, Odom GL. Intracranial metastases and their neurosurgical treatment. J Neurosurg 1965;23(6):375383.

Source of Support: Nil; Conflict of Interest: None

Submitted: 01-10-2018; Accepted: 14-10-2018; Published online: 13-11-2018 